\title{
Schneiderian membrane perforation via transcrestal sinus floor elevation: A randomized ex vivo study with endoscopic validation
}

\author{
Jordi Gargallo-Albiol ${ }^{1,2}$ (D) | Mustafa Tattan ${ }^{1}$ (D) | Khaled H. Sinjab ${ }^{1}$ | Hsun-Liang Chan ${ }^{1}$ | \\ Hom-Lay Wang ${ }^{1}$
}

${ }^{1}$ Department of Periodontics and Oral Medicine, University of Michigan School of Dentistry, Ann Arbor, Michigan

${ }^{2}$ Oral and Maxillo-facial Surgery Department, Universitat Internacional de Catalunya, Sant Cugat del Vallés, Barcelona, Spain

\section{Correspondence}

Jordi Gargallo-Albiol, Department of Periodontics and Oral Medicine, University of Michigan, Ann Arbor, MI.

Email: jgargallo@uic.es

Funding information

This paper was partially supported by the University of Michigan Periodontal Graduate Student Research Fund.

\begin{abstract}
Objective: To endoscopically determine the incidence of Schneiderian membrane perforation during transcrestal maxillary sinus floor elevation (SFE), in relation to the bone preparation technique, amount of bone graft, membrane elevation height and different surgical steps.

Materials and methods: Seven cadaver heads corresponding to 12 maxillary sinuses were used to perform three SFE via transcrestal approach per sinus (36 elevations). Each sinus was randomly assigned to either the Sinus Crestal Approach (SCA) drill kit technique (experimental group) or the conventional osteotome technique (control group). During all phases of the surgery, the integrity of the sinus membrane was monitored through endoscopic examination.

Results: A significant difference was found in the incidence of perforation $(p=0.007)$ and vertical elevation height $(p<0.001)$ between the study groups, favoring the experimental group. A safety elevation threshold of $5 \mathrm{~mm}$ without bone graft and implant placement was estimated. A significant correlation was observed between the residual ridge height and the incidence of perforation $(p<0.001 ; \mathrm{OR}=0.51)$.

Conclusion: The SCA drill kit may demonstrate superior osteotomy preparation and membrane elevation capabilities to the osteotome technique, and significantly when a 6-mm SFE is indicated. Residual ridge height and vertical elevation height are risk determinant factors.
\end{abstract}

\section{KEYWORDS}

bone substitutes, clinical assessment, diagnosis, sinus floor elevation

\section{1 | INTRODUCTION}

Expansion of the maxillary sinus and resorption of the residual ridge, following upper molar and premolar extraction, may compromise the dental implant placement in the maxillary posterior area. Maxillary sinus floor elevation (SFE), performed either via the lateral window or transcrestal approach, is usually indicated to overcome limitations in residual ridge height $(\mathrm{RRH}$; Boyne \& James,
1980). The transcrestal approach involves SFE with simultaneous placement of an implant (Summers, 1994; Tatum, 1986). The original procedure consists of inwardly fracturing the sinus floor by preparing the implant bed with osteotomes of increasing diameters. Other techniques were later proposed, such as the balloon technique (Chan et al., 2013; Yassin Alsabbagh, Alsabbagh, Darjazini Nahas, \& Rajih, 2017) and the piezotome technique, a procedure of standardized sequence of designed drills, trephine and osteotomes 
(Kim, Lee, Park, Kim, \& Oh, 2017; Trombelli, Franceschetti, Trisi, \& Farina, 2015).

The transcrestal approach is a less invasive, commonly applied technique for SFE. It's reportedly associated with increased patient acceptance and reduced patient discomfort when compared to the lateral window approach (Emmerich, Att, \& Stappert, 2005). The former is suitable where the relative residual ridge height is approximately 5-9 mm, exhibiting good long-term clinical outcomes and minimal complications (Katranji, Fotek, \& Wang, 2008; Lundgren et al., 2017; Pjetursson \& Lang, 2014). However, membrane perforation is a commonly occurring intraoperative complication, with prevalence up to $40 \%$ (Garbacea et al., 2012).

Schneiderian membrane perforation is often undetectable by the operator during surgical transcrestal SFE procedures, and this may impact the probability of postoperative complications. Antibiotic use for postoperative sinusitis, infection and bone graft failure was shown to be significantly higher in sinuses with perforated membranes (Nolan, Freeman, \& Kraut, 2014; Schwarz et al., 2015). A small perforation within the membrane may result in communication directly between the sinus cavity and the graft material. This can lead to infection and chronic sinusitis, which eventually result in the loss of the graft volume and/or implant failure (Katranji et al., 2008).

Detection of membrane perforations during and following transcrestal SFE is challenging. Therefore, efforts should be directed toward identifying the tools and factors associated with prevention, ultimately decreasing the likelihood of postoperative complications and improving treatment outcomes. Cone beam computed tomography $(C B C T)$ or periapical digital radiographs seem to be less precise than the endoscope for the detection of Schnederian membrane perforations in human cadaver investigations (Garbacea et al., 2012).

Hence, the primary aim of this ex vivo study was to endoscopically evaluate the incidence of Schneiderian membrane perforation associated with the different approaches to transcrestal SFE, namely SCA and osteotome techniques. The secondary aim was to assess the association of the membrane elevation height, amount of bone graft, residual ridge height and the different surgical steps on the incidence of perforation during a transcrestal SFE.

\section{2 | MATERIALS AND METHOD}

\section{1 | Study design}

Seven, fresh cadaver heads with fully or partially edentulous maxillary arches were provided by the Department of Anatomy at the University of Michigan. These specimens were frozen at a temperature of $-20^{\circ} \mathrm{C}$, after being harvested from human donors, to prevent structural changes in the tissues. Prior to being used in this study, the cadaver heads were completely thawed for a period of 4 to 5 days at room temperature. The University of Michigan (U-M) Institutional Review Board approved this study as exempt from oversight (HUM00138166).

The included heads corresponding to 12 maxillary sinuses (five bilateral maxillary sinuses with fully edentulous maxillary arches and two unilateral maxillary sinuses with partially edentulous maxillary arches) were used to perform three elevation procedures via the transcrestal approach per maxillary sinus (a total of 36 elevations). Each sinus was randomly assigned to receive a different elevation technique: Sinus Crestal Approach (SCA) drill kit (Neobiotech, Seoul, South Korea) (experimental group) or the osteotome technique (control group). The bilateral maxillary sinuses were used in a split-mouth manner, where the test was randomly assigned to one side and the control to the opposing side. Meanwhile, the two unilateral maxillary sinus heads were each randomly assigned to one of the two study groups. The randomization was performed by a specialized software (randomized.com, Shogun Interactive Development 2006); numbers 1 and 2 indicated the right side be experimental and control, respectively.

With 18 sinus elevations per study group, six mesial elevations consisted of membrane elevation only (without graft or implant placement) (subgroup A). Meanwhile, the remaining 12 elevations comprised six middle 3-mm (subgroup B) and six distal 6-mm (subgroup C) membrane elevations with bone graft insertion and subsequent implant placement.

\section{2 | Eligibility criteria}

The inclusion criteria comprised the following:

- Totally or partially edentulous posterior maxillary arches

- Absence of sinus pathology evident in the three-dimensional radiological assessment

- Sinuses with a relatively flat floor as indicated to be performed in the clinical basis

- Maxillary sinus free from sinus septa as pre-surgically examined using CBCT

Specimens were excluded if:

- The posterior maxillary arch was dentate, preventing access for a transcrestal sinus lift

- The posterior maxillary arch width was $<3 \mathrm{~mm}$

- The presence of a large sinus pathology was detected via the preprocedural CBCT imaging

\section{3 | CBCT data acquisition}

Tenting screws (Salvin Dental Specialties, Charlotte, NC, USA) were inserted bilaterally in each of the maxillary canine areas. These functioned as reference points, visible in the ments and identification of precise drilling sites to be made across the arch during the surgical procedure. The CBCT scans were obtained by a trained operator (KS) in the Radiology Department at the University of Michigan School of Dentistry. The specimens were stabilized using a head locator. Each maxillary sinus was examined pre-surgically in CBCT scans (3D Accuitomo 170 Tomograph, J 
Morita, Kyoto, Japan) with a voxel size of $0.08-0.16 \mathrm{~mm}$. The operating parameters were set at $5.0-7.0 \mathrm{~mA}$ and $90 \mathrm{kV}$. Exposure time was $17.5 \mathrm{~s}$. Limited FOV was selected for all images. The CBCT scans of each head were reconstructed with a built-in software and analyzed on a desktop computer with a specialized implant planning software (Invivo5; InvivoDent, Anatomage, San Jose, CA, USA). The СВCT images were evaluated by one author, an experienced oral surgeon (JG), on a desktop monitor (28-inch Dell 2407, resolution $1,920 \times 1,200$ pixels, refresh rate $59 \mathrm{~Hz}$; Dell Inc., Round Rock, TX, USA) under room lighting, and at a position of approximately $30 \mathrm{~cm}$ from the monitor. The CBCT images were reoriented to get (a) the nasal spine and midline aligned in the center of the image in the axial slice, (b) the posterior maxillary segment in the vertical position in the coronal slices and (c) the hard palate, as well as the floor of the nose in the horizontal position parallel to the ground in the sagittal slices. For the evaluation of intra-examiner reliability, all the measurements were performed twice at different days. The mean difference between the two measurements in bone parameters was $0.01 \mathrm{~mm}$ (range -0.059 to 0.079 ). For image assessment, each sample was conducted twice and a mean value was obtained (Janner et al., 2011). If $a \geq 0.2 \mathrm{~mm}$ difference was measured the same point, a third assessment was performed (Bornstein, Lauber, Sendi, \& von Arx, 2011; Froum, Khouly, Favero, \& Cho, 2013). Similarly, a second examiner (MT) randomly selected two cases to evaluate inter-examiner reliability, where a 0.86 Interclass Correlation coefficient was obtained, indicating near absolute agreement.

\subsection{Surgical procedure}

An experienced surgeon (JG) performed the surgical procedures taking into account the CBCT analysis and measurements. A middle crestal incision and a mesial vertical releasing incision above the canine area were performed to elevate a full thickness flap. The CBCT measurements made from the tenting screws to the planned drill sites were extrapolated to the surgical setup, where the same measurements were made onto the exposed bone (Figure 1). Bone preparation followed according to implant size, manufacturer guidelines and study group design. The depth of the preparation was determined based on the RRH measured on the CBCT images.

\section{5 | Sinus membrane elevation}

The experimental group osteotomies were performed using a series of increasing diameter SCA kit drills, connected with a stopper, according to the manufacturer's instructions (Figure 2a). Stoppers defined the drilling length according to the residual bone height and membrane elevation. In the control group, bone preparation and sinus membrane elevation were performed according to previous publications (Lundgren et al., 2017; Pjetursson \& Lang, 2014). Bone preparation to $1 \mathrm{~mm}$ below the sinus membrane was performed using the standard implant drilling protocol (Zimmer/Biomet 3i, West Palm Beach, FL, USA), starting from the pilot-drill (ø $2.3 \mathrm{~mm}$ ) to $3.4 \mathrm{~mm}$ of diameter, followed by passing with the osteotome to

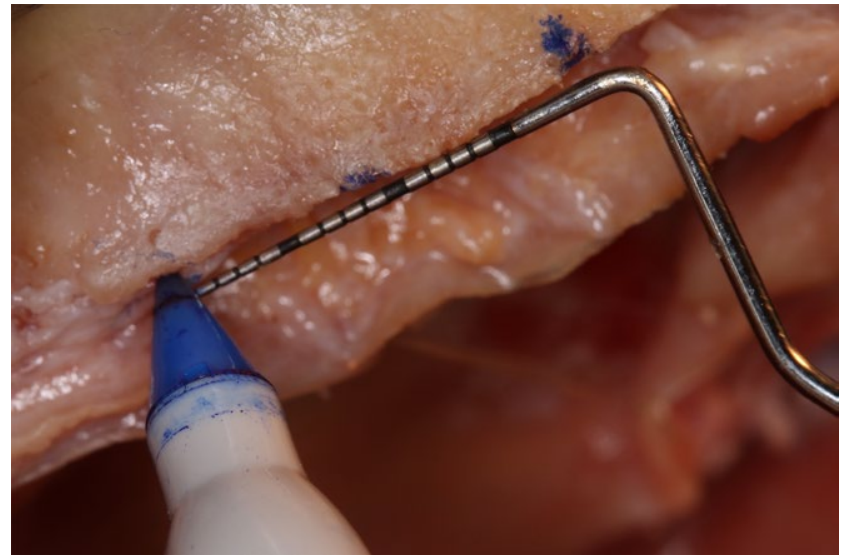

FIGURE 1 Bone marking measurements from the tenting screws to the planned drill sites

fracture the bony floor of the sinus and initiating membrane elevation. Initially, an osteotome of a small diameter and a light mallet were used to fracture the residual bone. Once bone preparation was complete, a second osteotome (ø 3.3 mm; Hu-Friedy, Chicago, IL, USA) was used to elevate the sinus membrane with precise control of the penetration length (Figure $2 b$ ).

The membrane's vertical elevation height (VEH) was measured using a calibrated gauge (Neobiotech, Seoul, South Korea). Sinus membrane elevation with or without bone graft and implant placement followed, according to the designed experimental workflow. The mesial elevation site was performed without bone graft or implant placement, to the heights of $3,4,5$, and $6 \mathrm{~mm}$ consecutively until perforation was achieved, at which point the final height was recorded. The middle and distal elevation sites were treated with bone graft insertion and implant placement to 3 and $6 \mathrm{~mm}$, respectively. Particulate allogenic bone graft (enCore Combination Allograft, particle size: 0.25-1 mm; Osteogenics Biomedical, Inc. Lubbock, TX, USA) was packed into the osteotomy site in the respective subgroups, before proceeding to implant placement. The amount of bone graft was measured prior to surgery using a scientific bascule (Mettler Toledo Balance AG204, Marshall Scientific, Hampton, NH, USA) to standardize the exact amount of graft material for each location:

1. $0.1 \mathrm{cc}$ of bone graft in the middle site to $3 \mathrm{~mm}$ of elevation.

2. $0.3 \mathrm{cc}$ of bone graft in the distal site to $6 \mathrm{~mm}$ of elevation.

Zimmer tapered screw vent dental implants (Zimmer/Biomet 3i) of $3.7 \mathrm{~mm} \times 13 \mathrm{~mm}$ dimensions were placed only to the desired preplanned height pertaining to each subgroup, when membrane perforation was not detected, in the middle and distal sites after bone graft was inserted.

\subsection{Data retrieval}

Residual bone height and width, and sinus membrane thickness were evaluated in each maxillary sinus and at each elevation site (mesial, middle and distal) in relation to the tenting screw reference 


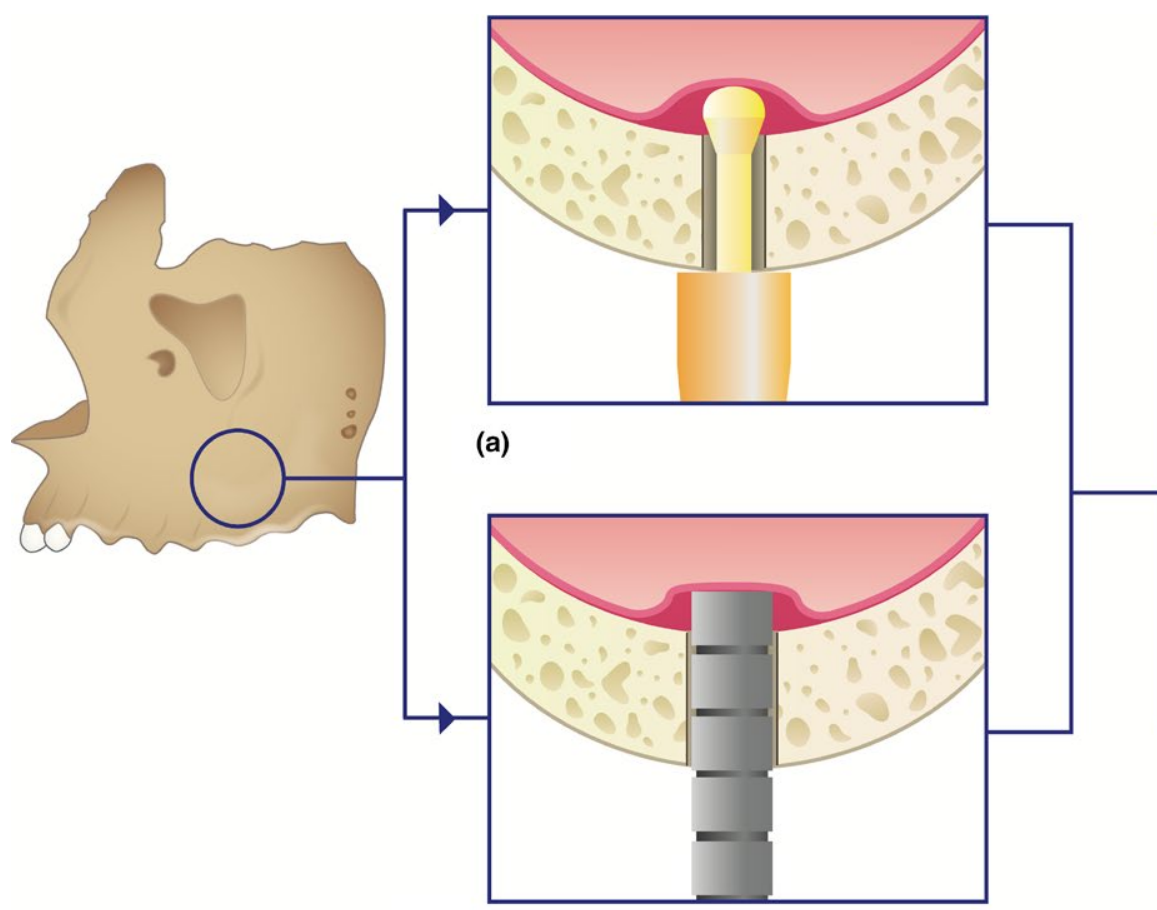

(b)

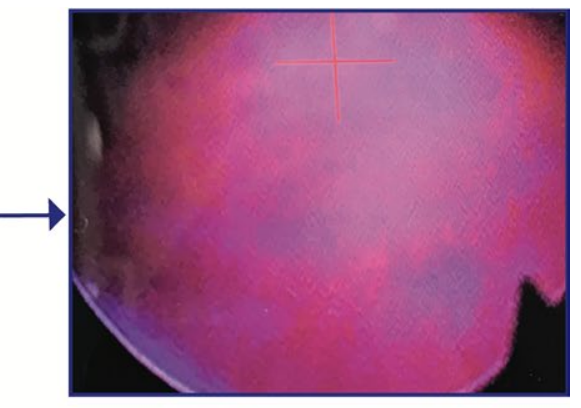

(c)

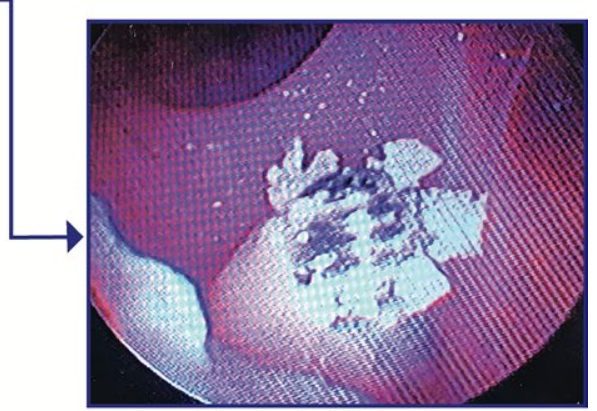

(d)

FIG URE 2 Bone preparation: (a) SCA drill kit (experimental group); (b) osteotome (control group); (c) Endoscopic image of sinus membrane elevation without perforation; (d) Endoscopic image of membrane perforation and direct communication of the implant and bone graft with sinus cavity

pre-surgically viewed in the CBCT scans. An endoscope (OTV-S5 Rhinolaryngoscope; Olympus, Center Valley, PA, USA), including an optical system allowing for $90^{\circ}$ field of view and 5-50 mm depth of field, was used by a single investigator (MT) to monitor the sinus membrane perforation during the sinus elevation procedures. The insertion tube was $3.2 \mathrm{~mm}$ in diameter and possesses a $130^{\circ} \mathrm{up} /$ down bending capability. A fiber light projector (Richard Wolf model 5119 USA Medical Instruments Corp. Vernon Hills, IL, USA) was used in combination with the fiberscope. Intra-surgical images were obtained and transferred to a processor that displays the visual across a connected monitor. For efficiency of time, the endoscope was inserted before starting the STE surgery into the sinus via an opening $(10 \times 5 \mathrm{~mm})$ below the inferior orbital rim. At that point, the sinus membrane was exposed and checked for complete defrosting. The endoscope images were monitored by one investigator (MT) during the elevation procedure and the effect of bone graft insertion and implant placement on the membrane integrity was constantly monitored (Figure $2 \mathrm{c}$ and d). The second investigator was instructed to adjourn the procedure when a perforation was visually detected.

\section{7 | Measurements}

The procedure was deemed successful when the membrane was elevated without perforation; otherwise, it was considered a failure. The elevation was measured (in $\mathrm{mm}$ ) from the alveolar crest to the topmost point. The VEH was calculated as the final membrane height minus the RRH. The BPE (Bucco-palatal elevation) was the measured on a cross-sectional slice of the CBCT image (Figure 3a), while MDE (Mesio-distal elevation) was measured on a sagittal slice of the image (Figure $3 \mathrm{~b}$ ).

Cone beam computed tomographies before and after the surgery were obtained and a continuous endoscopy procedure, to check the integrity of the sinus membrane, was monitored during the surgery. All the following variables/measurements were obtained and recorded at each of the three elevation sites per sinus, amounting to 36 sites:

- Residual ridge height (RRH) (mm)

- Residual ridge width (RRW) (mm)

- Membrane Thickness (MT) (mm)

- Vertical elevation height (VEH) (mm)

- Bucco-palatal elevation (BPE) (mm)

- Mesio-distal elevation (MDE) (mm)

- The VEH to BPE ratio (VEH:BPE)

- The VEH to MDE ratio (VEH:MDE)

- Incidence of Perforation (IoP) (1-0)

- Implant Placement (IP) (1-0)

- Volume of bone graft inserted (cc)

- Stage of membrane perforation: bone preparation, bone grafting or implant placement

\subsection{Statistical analysis}

Statistical analysis was expressed using the mean, minimum and maximum values, and standard deviations (SD). Generalized estimating 
FIGURE 3 Cone beam Computed Tomography of sinus membrane elevation with the vertical elevation height: (a) bucco-palatal elevation; (b) mesio-distal elevation

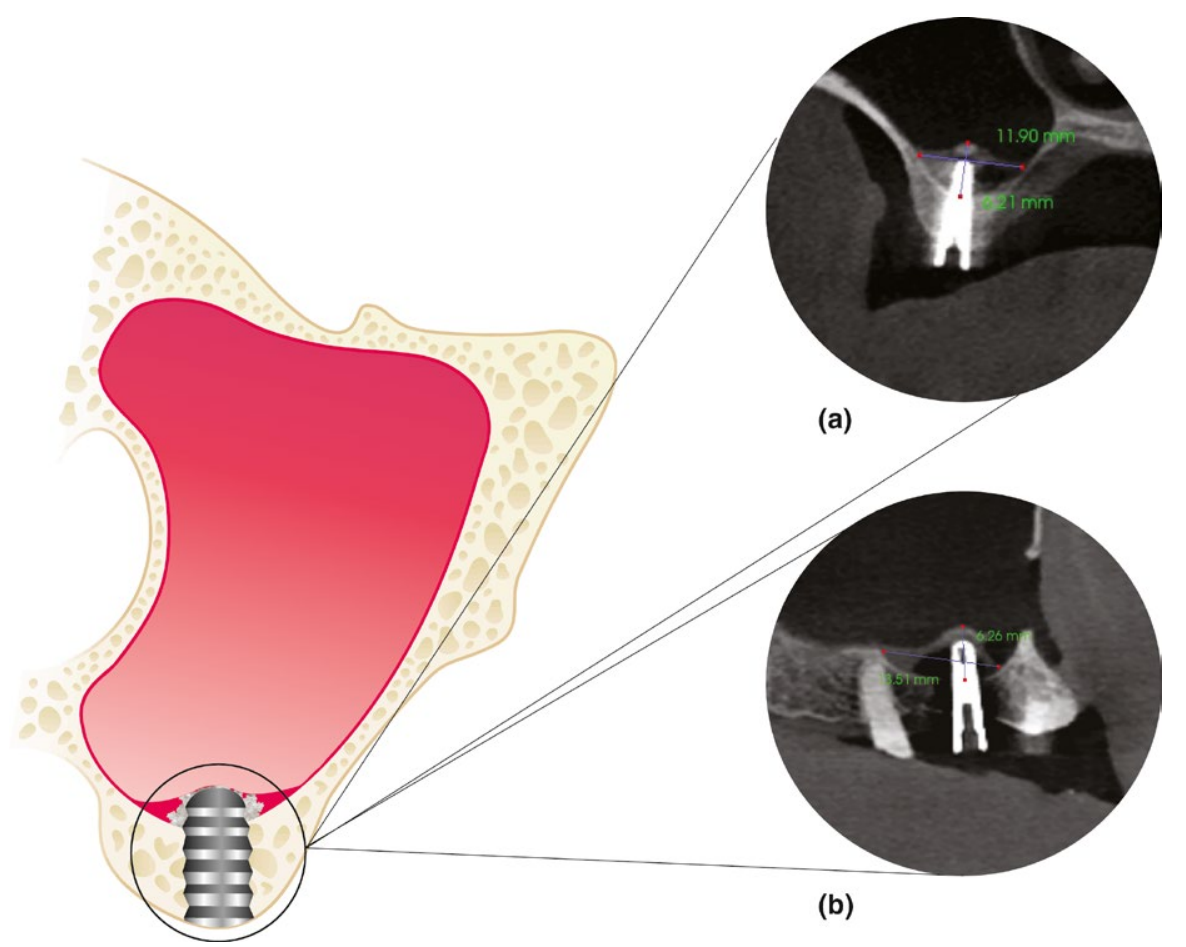

equations (GEE) methods were used to test the effect of the elevation technique, MT, RRH, bone graft, VEH, VEH:BPE and VEH:MDE on the dependent variable loP. Non-adjusted and adjusted odds ratio (OR) and $95 \%$ confidence intervals were obtained from univariate and multivariate binary logistic regressions using GEE to consider the clustered structure of the data. GEE linear models were also used to study the differences of the membrane thickness or VEH between the groups.

The significance level was defined as $p<0.05$, for all statistical tests. In all statistical tests involving the study groups variable, the experimental and control groups were considered groups 1 and 2 , respectively. All the analyses were conducted with a specialized software package (IBM SPSS Statistics 24, Armonk, NY, USA).

\section{RESULTS}

\section{1 | Descriptive analysis}

A total of seven unfixed, fresh cadaver heads (four males and three females), with 10 bilateral and two unilateral maxillary sinuses, qualified to be included in the study. The reason for exclusion of two unilateral sinuses was a result of corresponding to the dentate ridges, while all the included sinuses corresponded to fully or partially edentulous ridges. A total of 36 transcrestal SFE procedures were performed. The mean ridge width in the study sample was $7.13 \pm 1.56 \mathrm{~mm}$. A complete descriptive analysis of the data based on the study groups and subgroups is summarized in Table 1.

\subsection{Schneiderian membrane thickness}

The data of six sites were excluded from only the CBCT membrane measurements due to image artifacts/distortion that interfere with the accurate analysis at the region of interest. Of these excluded sites, three belonged to subgroup A (resultant $n=9$ ), two belonged to subgroup $B$ (resultant $n=10$ ) and one belonged to subgroup $C$ (resultant $n=11$ ). Thus, a total of 30 membrane thickness measurements were obtained from the seven included heads. The mean membrane thickness in the total study sample was $0.93 \pm 0.66 \mathrm{~mm}$ $(0.39-2.91 \mathrm{~mm})$, with no statistically significant difference between the subgroups $(p=0.264)$.

\section{3 | Incidence of Schneiderian membrane perforation}

The percentage of loP in the entire study sample was 50\% (33\% in the experimental group and $66.7 \%$ in the control group) (Table 1). This difference, between the two groups, was not found to be statistically significant $(\mathrm{OR}=0.25 ; p=0.138)$. However, when the model was adjusted by other independent variables (RRH, BG), significance was reached $(\mathrm{OR}=0.04 ; p=0.007)$. A similar conclusion was obtained from the adjustment by $\mathrm{VEH}$ and ratios (OR $=0.02 ; p=0.046)$.

Within the perforations seen in subgroups $A, B$ and $C$, a total of $16.7 \%, 50 \%$ and $33.3 \%$ belonged to the experimental group, respectively. Although no significant difference in loP comparing the three subgroups was observed, all the perforations in subgroup A were at $6 \mathrm{~mm}$ of VEH. This demonstrates an estimated maximum safety elevation threshold of $5 \mathrm{~mm}$ without bone graft and implant placement.

\subsection{Associated variables of the Schneiderian membrane perforation}

The GEE model demonstrated a statistically significant negative correlation between IoP and RRH $(p<0.001$; OR $=0.51)$ and a lack of 
TABLE 1 Demographics of the sinus elevation variables

\begin{tabular}{|c|c|c|c|c|c|c|c|c|c|c|c|}
\hline & \multicolumn{3}{|c|}{ loP } & \multicolumn{2}{|c|}{$\mathrm{RRH}(\mathrm{mm})$} & \multicolumn{2}{|c|}{ VEH (mm) } & \multicolumn{2}{|c|}{ VEH:BPE } & \multicolumn{2}{|c|}{ VEH:MDE } \\
\hline Test & 18 & 6 & 33 & 4.65 & 2.24 & 6.32 & 1.00 & 0.77 & 0.15 & 0.76 & 0.26 \\
\hline Control & 18 & 12 & 66.7 & 6.72 & 2.53 & 3.71 & 0.86 & 0.45 & 0.12 & 0.50 & 0.10 \\
\hline A & 12 & 6 & 50 & 7.02 & 2.41 & N/A & & N/A & & N/A & \\
\hline
\end{tabular}

Note. BPE: bucco-palatal elevation; IoP: incidence of perforation; MDE: mesio-distal elevation; RRH: residual ridge height; VEH: vertical elevation height.

correlation between IoP and the amount of bone graft ( $p=0.229$; Table 2: model n.2). The mean RRH associated with perforations and non-perforations in the experimental group was $3.18 \mathrm{~mm} \pm 1.73$ and $5.38 \mathrm{~mm} \pm 2.16$, respectively. Contrarily, the mean RRH associated with perforations and non-perforations in the control group was $5.89 \mathrm{~mm} \pm 1.82$ and $8.40 \mathrm{~mm} \pm 3.07$, respectively. $\mathrm{RRH}$ associated with perforation was concluded as significantly lower in the test group $(p<0.001)$.

Also outlined in Table 2, the regression model 3 analyzing loP with $\mathrm{VEH}$, the ratio of $\mathrm{VEH}$ to BPE and the ratio of VEH to MDE indicated a significant positive correlation between IoP and VEH $(p=0.004$; $\mathrm{OR}=3.47$ ). The correlation was also positive with $\mathrm{VEH}: \mathrm{BPE}$ and negative with $\mathrm{VEH}: M D E$, but statistical significance was not reached ( $p=0.613, p=0.525$ respectively). This indicates that with increased $\mathrm{VEH}$, the probability of loP is expected to also increase.

Wald's $\mathrm{Chi}^{2}$ from GEE model determined a significant degree of variance between the $\mathrm{VEH}$ in the experimental group versus the control group $(p<0.001)$. This indicates that significantly more VEH was permitted in the experimental group, as opposed to the control group. However, no such significance was observed in terms of implant placement ( $p=0.277$; Table 3).

Finally, with regard to loP relative to the stage of surgery, 15 (83.3\%) of the membrane perforations were found to have occurred during the first (elevation) phase of surgery, while 0 and 3 (16.7\%) of the membrane perforations occurred during the second (bone graft insertion) and third (implant placement; Figure 2d) phases of surgery, respectively. This difference was statistically significant $(p=0.005)$.

\section{DISCUSSION}

Schneiderian membrane perforation is one of the most critical challenges of maxillary SFE and is associated with a higher prevalence of postoperative sinusitis (Schwarz et al., 2015). The percentage of perforations found in the present study was $50 \%$, higher than the data reported by Garbacea et al. (2012) and Nolan et al. (2014), who reported a mean loP rate of $40 \%$ and $41 \%$, respectively. These rates were considerably less than the $58.4 \%$ reported by Yassin Alsabbagh et al. (2017), or the $62.5 \%$ reported by Cho, Wallace, Froum, and Tarnow (2001). However, perforation during transcrestal sinus membrane elevation is not always detected, indirectly impacting postoperative complications and surgical outcome. If this occurs, a number of consequences may entail: the presence of bone graft within the sinus antrum, acute or chronic sinus infection, the invasion of bacteria into the site or disrupted maxillary sinus physiologic function (Katranji et al., 2008; Li \& Wang, 2008). With all the proposed methods of crestal SFE (Chan et al., 2013; Kim et al., 2017; Yassin Alsabbagh et al., 2017), MT (Wen, Lin, Yang, \& Wang, 2015) RRH (Schwarz et al., 2015), the amount of bone graft inserted and VEH (Sonoda, Harada, Yamamichi, Monje, \& Wang, 2017) are factors that influence the probability of membrane perforation. In this study sample, MT was not statistically significant between the two study groups, enabling a fair comparison of all other factors between test and control.

Schneiderian membrane elevation with SCA drill kit has the advantage of using a reamer to create the osteotomy in a conical shape and break the bony floor avoiding damage to the sinus membrane. However, it is important to note that although the SCA kit has shown to be superior to the osteotome technique in the present investigation, this difference was only statistically significant in subgroup $C$, where the elevation was beyond $3 \mathrm{~mm}$. Thus, it can be deduced that the two techniques are comparable when minimal elevation is necessary; however, when more than $3 \mathrm{~mm}$ and up to $6 \mathrm{~mm}$ of elevation is indicated, the SCA kit maintains membrane integrity significantly better. This could be explained by the greater VEH permitted by the SCA versus osteotome approach. The positive results attributed to this kit demonstrated in this study are in concordance with the results observed in the ex vivo study of Yassin Alsabbagh et al., 2017, where the SCA drill kit was shown to be superior to the osteotome technique in osteotomy preparation and breaking the sinus floor. These results were later corroborated in a clinical study by Kim et al., 2017, who did not report any membrane perforations using the SCA drill kit, but reported an incident of acute maxillary sinusitis 5 months after surgery. This may have been related to a possible undetected perforation during the elevation surgery. On the other hand, according to a dentists' subjective satisfaction survey performed following maxillary sinus membrane elevation via the crestal approach, $92.9 \%$ of the dentists were generally satisfied with the SCA approach to elevate the membrane instead of the hydraulic approach (Kim, Yang, Chung, Kim, \& Yeo, 2013). 
TAB LE 2 Univariate (model 1) and multivariate (model 2 and 3 ) logistic regression (GEE) models analyzing the association between the incidence of perforation (IoP) and other variables of elevation, namely: study groups, residual ridge height ( $R R H)$, bone graft (BG), vertical elevation height (VEH), VEH:bucco-palatal elevation ratio (VEH:BPE) and VEH:mesio-distal elevation ratio (VEH:MDE). VEH:MDE was excluded from model 3 because of a lack of convergence of GEE estimations

\begin{tabular}{|c|c|c|c|c|c|}
\hline \multirow[b]{2}{*}{ Parameter } & \multirow[b]{2}{*}{$\beta$} & \multirow[b]{2}{*}{ OR } & \multicolumn{2}{|c|}{$\begin{array}{l}95 \% \text { Wald confidence } \\
\text { interval for OR }\end{array}$} & \multirow{2}{*}{$\begin{array}{l}\text { Hypothesis test } \\
p \text {-Value }\end{array}$} \\
\hline & & & Lower & Upper & \\
\hline \multicolumn{6}{|l|}{ Univariate model 1} \\
\hline Experimental group & -1.39 & 0.25 & 0.04 & 1.56 & 0.138 \\
\hline $\mathrm{RRH}$ & -0.23 & 0.79 & 0.64 & 0.98 & 0.036 \\
\hline BG & 0.01 & 1.00 & 0.05 & 20.6 & 1.000 \\
\hline VEH & 0.28 & 1.32 & 0.44 & 3.92 & 0.619 \\
\hline VEH:BPE & 0.44 & 1.55 & 0.01 & 1,277 & 0.898 \\
\hline VEH:MDE & -2.02 & 0.13 & 0.01 & 67.1 & 0.525 \\
\hline \multicolumn{6}{|l|}{ Multivariate model 2} \\
\hline Experimental group & -3.32 & 0.04 & 0.01 & 0.41 & 0.007 \\
\hline RRH & -0.67 & 0.51 & 0.37 & 0.70 & $<0.001$ \\
\hline BG & -4.29 & 0.01 & 0.00 & 14.9 & 0.229 \\
\hline \multicolumn{6}{|l|}{ Multivariate model 3} \\
\hline Experimental group & -4.13 & 0.02 & 0.01 & 0.93 & 0.046 \\
\hline VEH & 1.25 & 3.47 & 1.50 & 8.05 & 0.004 \\
\hline VEH:BPE & 1.78 & 5.91 & 0.01 & 576.6 & 0.613 \\
\hline VEH:MDE & - & - & - & - & - \\
\hline
\end{tabular}

TAB LE 3 GEE model analyzing the association between the elevation technique and the implant placement (IP), as well as the vertical elevation height (VEH)

\begin{tabular}{|c|c|c|c|c|}
\hline & \multicolumn{4}{|c|}{$95 \%$ Wald confidence interval for $\beta$} \\
\hline & $\beta$ & $p$-Value & Lower & Upper \\
\hline \multicolumn{5}{|c|}{ Study groups (Experimental/Control) } \\
\hline VEH & 2.61 & $<0.001$ & 1.91 & 3.31 \\
\hline IP & 0.80 & 0.277 & 0.53 & 9.44 \\
\hline
\end{tabular}

The RRH has also been described as an influencing factor that impacts membrane perforation using the transcrestal approach (Schwarz et al., 2015), where a minimal RRH of $5 \mathrm{~mm}$ is recommended (Lundgren et al., 2017; Pjetursson \& Lang, 2014). These data support our results, where the RRH has been revealed as a statistically significant factor of membrane perforation. Schwarz et al., 2015 showed that RRH less than $3.5 \mathrm{~mm}$ was a main risk factor increasing the loP, in agreement with the $3.18 \mathrm{~mm} \pm 1.73$ mean $\mathrm{RRH}$ associated with perforations in the experimental group observed in this study.

Most of the membrane perforations within the non-grafted subgroup (A) were obtained when the height of elevation reached $6 \mathrm{~mm}$, meaning that below $6 \mathrm{~mm}$ represents a safe zone. Therefore, our results show the estimated $5-\mathrm{mm}$ elevation height as a safe zone, prior to bone graft insertion and implant placement. These findings come in line with Lundgren et al., 2017, who described that the elevation height in transcrestal SFE should not exceed 3-4 mm.

As reported, the amount of bone graft inserted has been related to the millimeters of sinus membrane vertically elevated, obtaining elevation heights of $3 \mathrm{~mm}$ or $6 \mathrm{~mm}$ when using 0.1 or $0.3 \mathrm{cc}$ of bone graft, respectively (Sonoda et al., 2017). Although the findings of this study showed that $\mathrm{VEH}$ plays a determinant role in maintaining sinus membrane integrity, the amount of bone graft inserted was not determinant. On the other hand, the VEH:BPE and VEH:MDE displayed a different impact on membrane perforation, demonstrating that mesio-distal (MD) augmentation in relation to VEH had a positive effect on membrane perforation. Contrary to this, bucco-palatal (BP) augmentation in relation to $\mathrm{VEH}$ appeared to have a negative effect on maintaining membrane integrity. These ratios describe the degree of three-dimensional horizontal extension as opposed to only the vertical extension of a SFE. It is critical to address the elevation procedure from all possible directions to better understand the tension distribution to be achieved. The findings in this study compliment those from Sonoda et al. (2017), who concluded that VEH:BPE and VEH:MDE should be $\leq 0.8$ to avoid sinus membrane perforation. From a clinical standpoint, VEH:BPE and VEH:MDE may be difficult factors to control in sinus membrane elevation via the transcrestal approach in comparison to the more invasive lateral approach, where the MD and BP bone augmentation could more practically be controlled. However, this effect requires further investigation.

According to Garbacea et al., 2012, Schneiderian membrane perforation during transcrestal sinus elevation can occur during different treatment stages: bone preparation/breaking the bony sinus floor, membrane elevation, graft insertion or implant placement. Hence, due to often being undetectable, special care is recommended during the membrane elevation phase and during the implant placement phase. With regard to implant placement, the implant may exert pressure on the bone graft that manages to perforate the sinus membrane, providing the bone graft with an escape 
route into the sinus cavity. However, no perforations were detected during the bone graft insertion phase in the present study, indicating that excessive perforation-inducing pressure is not exerted on the sinus membrane during bone graft insertion.

Despite the associated variables that may have an impact on the success or failure of the transcrestal SFE procedure, it must be noted that a sufficient amount of experience is a clear prerequisite. Both the technical approaches discussed in this study equally require adequate expertise prior to being performed successfully.

They are several limitations of this study, one of them is using cadavers to conduct this study; hence, the cadaver bone quality and membrane elasticity may differ from the living bone. To minimize the bias from specimen quality, we chose frozen fresh cadaver heads that have the most similar tissue situation to the actual human. Nonetheless, this remains a concern in the study. Additionally, since this is a cadaver study, we did not conduct the power calculation to determine the proper sample size. We only used available fresh cadaver heads that qualified to the study in conducting this investigation. Hence, limited sample size and lack of power calculation are the limitations noted in the study.

\section{5 | CONCLUSION}

The SCA drill kit may demonstrate superior osteotomy preparation and SFE capabilities to the osteotome technique, while both techniques require operator experience. This enhanced ability in elevation was especially significant when a 6-mm elevation was indicated. Also, a maximum safety elevation threshold of $5 \mathrm{~mm}$ without bone graft and implant placement was estimated. Finally, residual ridge height and vertical elevation height are the important factors of membrane perforation.

\section{ACKNOWLEDGEMENTS}

The authors would like to thank James Sugai for his help with the Giannobile lab facilities, and Oliver Kripfgans from the Radiology Department at the University of Michigan Hospital for his contribution with the endoscope and fiber light projector. Moreover, the authors want to express their gratitude to Alberto Monje (Department of Oral Surgery and Stomatology, University of Bern, Bern, Switzerland) for his assistance during the elaboration of this manuscript.

\section{CONFLICT OF INTEREST}

The authors do not have any financial interests, neither directly nor indirectly, in the products or information listed in the paper.

\section{ORCID}

Jordi Gargallo-Albiol iD https://orcid.org/0000-0002-9307-8258
Mustafa Tattan iD https://orcid.org/0000-0001-7498-8064

Hom-Lay Wang iD https://orcid.org/0000-0003-4238-1799

\section{REFERENCES}

Bornstein, M. M., Lauber, R., Sendi, P., \& von Arx, T. (2011). Comparison of periapical radiography and limited cone-beam computed tomography in mandibular molars for analysis of anatomical landmarks before apical surgery. Journal of Endodontics, 37(2), 151-157. https:// doi.org/10.1016/j.joen.2010.11.014

Boyne, P. J., \& James, R. A. (1980). Grafting of the maxillary sinus floor with autogenous marrow and bone. Journal of Oral and Maxillofacial Surgery, 38(8), 613-616.

Chan, H. L., Oh, T. J., Fu, J. H., Benavides, E., Avila-Ortiz, G., \& Wang, H. L. (2013). Sinus augmentation via transcrestal approach: A comparison between the balloon and osteotome technique in a cadaver study. Clinical Oral Implants Research, 24(9), 985-990. https://doi. org/10.1111/j.1600-0501.2012.02506.x

Cho, S. C., Wallace, S. S., Froum, S. J., \& Tarnow, D. P. (2001). Influence of anatomy on Schneiderian membrane perforations during sinus elevation surgery: Three-dimensional analysis. Practical Procedures \& Aesthetic Dentistry, 13(2), 160-163.

Emmerich, D., Att, W., \& Stappert, C. (2005). Sinus floor elevation using osteotomes: A systematic review and meta-analysis. Journal of Periodontology, 76(8), 1237-1251. https://doi.org/10.1902/ jop.2005.76.8.1237

Froum, S. J., Khouly, I., Favero, G., \& Cho, S. C. (2013). Effect of maxillary sinus membrane perforation on vital bone formation and implant survival: A retrospective study. Journal of Periodontology, 84(8), 1094-1099. https://doi.org/10.1902/jop.2012.120458

Garbacea, A., Lozada, J. L., Church, C. A., Al-Ardah, A. J., Seiberling, K. A., Naylor, W. P., \& Chen, J.-W. (2012). The incidence of maxillary sinus membrane perforation during endoscopically assessed crestal sinus floor elevation: A pilot study. The Journal of Oral Implantology, 38(4), 345-359. https://doi.org/10.1563/ aaid-joi-d-12-00083

Janner, S. F., Caversaccio, M. D., Dubach, P., Sendi, P., Buser, D., \& Bornstein, M. M. (2011). Characteristics and dimensions of the Schneiderian membrane: A radiographic analysis using cone beam computed tomography in patients referred for dental implant surgery in the posterior maxilla. Clinical Oral Implants Research, 22(12), 1446-1453. https://doi.org/10.1111/j.1600-0501.2010.02140.x

Katranji, A., Fotek, P., \& Wang, H. L. (2008). Sinus augmentation complications: Etiology and treatment. Implant Dentistry, 17(3), 339-349. https://doi.org/10.1097/ID.0b013e3181815660

Kim, H. Y., Yang, J. Y., Chung, B. Y., Kim, J. C., \& Yeo, I. S. (2013). Peri-implant bone length changes and survival rates of implants penetrating the sinus membrane at the posterior maxilla in patients with limited vertical bone height. Journal of Periodontal \& Implant Science, 43(2), 58-63. https://doi.org/10.5051/jpis.2013.43.2.58

Kim, Y. K., Lee, J. Y., Park, J. W., Kim, S. G., \& Oh, J. S. (2017). Sinus membrane elevation by the crestal approach using a novel drilling system. Implant Dentistry, 26(3), 351-356. https://doi.org/10.1097/ ID.0000000000000570

Li, J., \& Wang, H. L. (2008). Common implant-related advanced bone grafting complications: Classification, etiology, and management. Implant Dentistry, 17(4), 389-401. https://doi.org/10.1097/ ID.0b013e31818c4992

Lundgren, S., Cricchio, G., Hallman, M., Jungner, M., Rasmusson, L., \& Sennerby, L. (2017). Sinus floor elevation procedures to enable implant placement and integration: techniques, biological aspects and clinical outcomes. Periodontology 2000, 73(1), 103-120. https://doi. org/10.1111/prd.12165 
Nolan, P. J., Freeman, K., \& Kraut, R. A. (2014). Correlation between Schneiderian membrane perforation and sinus lift graft outcome: A retrospective evaluation of 359 augmented sinus. Journal of Oral and Maxillofacial Surgery, 72(1), 47-52. https://doi.org/10.1016/j. joms.2013.07.020

Pjetursson, B. E., \& Lang, N. P. (2014). Sinus floor elevation utilizing the transalveolar approach. Periodontology 2000, 66(1), 59-71. https:// doi.org/10.1111/prd.12043

Schwarz, L., Schiebel, V., Hof, M., Ulm, C., Watzek, G., \& Pommer, B. (2015). Risk factors of membrane perforation and postoperative complications in sinus floor elevation surgery: Review of 407 augmentation procedures. Journal of Oral and Maxillofacial Surgery, 73(7), 1275-1282. https://doi.org/10.1016/j.joms.2015.01.039

Sonoda, T., Harada, T., Yamamichi, N., Monje, A., \& Wang, H. L. (2017). Association between bone graft volume and maxillary sinus membrane elevation height. International Journal of Oral and Maxillofacial Implants, 32(4), 735-740. https://doi.org/10.11607/jomi.5290

Summers, R. B. (1994). A new concept in maxillary implant surgery: The osteotome technique. Compendium, 15(2), 152, 154-156, 158 passim; quiz 162.

Tatum, H. Jr (1986). Maxillary and sinus implant reconstructions. Dental Clinics of North America, 30(2), 207-229.

Trombelli, L., Franceschetti, G., Trisi, P., \& Farina, R. (2015). Incremental, transcrestal sinus floor elevation with a minimally invasive technique in the rehabilitation of severe maxillary atrophy. Clinical and histological findings from a proof-of-concept case series. Journal of Oral and Maxillofacial Surgery, 73(5), 861-888. https://doi.org/10.1016/j. joms.2014.12.009

Wen, S. C., Lin, Y. H., Yang, Y. C., \& Wang, H. L. (2015). The influence of sinus membrane thickness upon membrane perforation during transcrestal sinus lift procedure. Clinical Oral Implants Research, 26(10), 1158-1164. https://doi.org/10.1111/clr.12429

Yassin Alsabbagh, A., Alsabbagh, M. M., Darjazini Nahas, B., \& Rajih, S. (2017). Comparison of three different methods of internal sinus lifting for elevation heights of $7 \mathrm{~mm}$ : An ex vivo study. International Journal of Implant Dentistry, 3(1), 40. https://doi.org/10.1186/ s40729-017-0103-5

\section{SUPPORTING INFORMATION}

Additional supporting information may be found online in the Supporting Information section at the end of the article.

How to cite this article: Gargallo-Albiol J, Tattan M, Sinjab KH, Chan $\mathrm{H}$-L, Wang $\mathrm{H}$-L. Schneiderian membrane perforation via transcrestal sinus floor elevation: A randomized ex vivo study with endoscopic validation. Clin Oral Impl Res. 2019;30:11-19. https://doi.org/10.1111/clr.13388 